\title{
On nonlocal three-point boundary value problems of Duffing equation with mixed nonlinear forcing terms
}

Ahmed Alsaedi ${ }^{*}$ and Mohammed HA Aqlan

\author{
* Correspondence: \\ aalsaedi@hotmail.com \\ Department of Mathematics, \\ Faculty of Science, King Abdulaziz \\ University, P.O. Box. 80203, Jeddah \\ 21589, Saudi Arabia
}

\begin{abstract}
In this paper, we investigate the existence and approximation of the solutions of a nonlinear nonlocal three-point boundary value problem involving the forced Duffing equation with mixed nonlinearities. Our main tool of the study is the generalized quasilinearization method due to Lakshmikantham. Some illustrative examples are also presented.

Mathematics Subject Classification (2000): 34B10, 34B15.

Keywords: Duffing equation, nonlocal boundary value problem, quasilinearization, quadratic convergence
\end{abstract}

\section{Introduction}

The Duffing equation plays an important role in the study of mechanical systems. There are multiple forms of the Duffing equation, ranging from dampening to forcing terms. This equation possesses the qualities of a simple harmonic oscillator, a nonlinear oscillator, and has indeed an ability to exhibit chaotic behavior. Chaos can be defined as disorder and confusion. In physics, chaos is defined as behavior so unpredictable as to appear random, allowing great sensitivity to small initial conditions. The chaotic behavior can emerge in a system as simple as the logistic map. In that case, the "route to chaos" is called period-doubling. In practice, one would like to understand the route to chaos in systems described by partial differential equations such as flow in a randomly stirred fluid. This is, however, very complicated and difficult to treat either analytically or numerically. The Duffing equation is found to be an appropriate candidate for describing chaos in dynamic systems. The advantage of a pseudochaotic equation like the Duffing equation is that it allows control of the amount of chaos it exhibits. Chaotic oscillators are important tools for creating and testing models that are more realistic. This is why the Duffing equation is of great interest. The use of the Duffing equation aids in the dynamic behavior of chaos and bifurcation, which studies how small changes in a function can cause a sudden change in behavior [1]. Another important application of the Duffing equation is in the field of the prediction of diseases. A careful measurement and analysis of a strongly chaotic voice has the potential to serve as an early warning system for more serious chaos and possible onset of disease. This chaos is with the help of the Duffing equation. In fact, the

(C) 2011 Alsaedi and Aqlan; licensee Springer. This is an Open Access article distributed under the terms of the Creative Commons Attribution License (http://creativecommons.org/licenses/by/2.0), which permits unrestricted use, distribution, and reproduction in any medium, provided the original work is properly cited. 
success at analyzing and predicting the onset of chaos in speech and its simulation by equations such as the Duffing equation has enhanced the hope that we might be able to predict the onset of arrhythmia and heart attacks someday [2].

The Duffing equation is a mathematical representation of the oscillator. Both the equation and oscillator are prone to many output waveforms. One of the simplest waveforms includes simple harmonic motion like a pendulum. Other waveforms are considerably more complex and can quickly be described as shear oscillatory chaos. The Duffing equation can be a forced or unforced damped chaotic harmonic oscillator. Exact solutions of second-order nonlinear differential equations like the forced Duffing equation are rarely possible due to the possible chaotic output. There do exist a number of powerful procedures for obtaining approximate solutions of nonlinear problems such as Galerkin's method, expansion methods, dynamic programming, iterative techniques, the method of upper and lower bounds, and Chapligin method to name a few. The monotone iterative technique coupled with the method of upper and lower solutions [3] manifests itself as an effective and flexible mechanism that offers theoretical as well as constructive existence results in a closed set, generated by the lower and upper solutions. In general, the convergence of the sequence of approximate solutions given by the monotone iterative technique is at most linear. To obtain a sequence of approximate solutions converging quadratically, we use the method of quasilinearization. The origin of the quasilinearization lies in the theory of dynamic programming $[4,5]$. Agarwal [6] discussed quasilinearization and approximate quasilinearization for multipoint boundary value problems. In fact, the quasilinearization technique is a variant of Newton's method. This method applies to semilinear equations with convex (concave) nonlinearities and generates a monotone scheme whose iterates converge quadratically to a solution of the problem at hand. The nineties brought new dimensions to this technique when Lakshmikantham $[7,8]$ generalized the method of quasilinearization by relaxing the convexity assumption. This development was so significant that it attracted the attention of many researchers, and the method was extensively developed and applied to a wide range of initial and boundary value problems for different types of differential equations. A detailed description of the quasilinearization method and its applications can be found in the monograph [9] and the papers [10-26] and the references therein.

In this paper, we study a nonlinear nonlocal three-point boundary value problem of the forced Duffing equation with mixed nonlinearities given by

$$
\begin{aligned}
& x^{\prime \prime}(t)+\lambda x^{\prime}(t)=N(t, x(t)), \quad t \in J=[0,1], \quad \lambda \in \mathbb{R}-\{0\}, \\
& p x(0)-q x^{\prime}(0)=g_{1}(x(\sigma)), \quad p x(1)+q x^{\prime}(1)=g_{2}(x(\sigma)), \quad 0<\sigma<1, \quad p, q>0,
\end{aligned}
$$

where $N(t, x) \in C[\times \mathbb{R}, \mathbb{R}]$ is such that

$$
N(t, x)=f(t, x)+k(t, x)+H(t, x),
$$

and $g_{i}: \mathbb{R} \rightarrow \mathbb{R}(i=1,2)$ are given continuous functions. The details of such a decomposition can be found in Section 1.5 of the text [9]. In (1.3), it is assumed that $f(t, x)$ is nonconvex, $k(t, x)$ is nonconcave, and $H(t, x)$ is a Lipschitz function:

$$
H(t, x)-H(t, y) \geq-L(x-y), x \geq y, x, y \in \mathbb{R}, L>0 .
$$


A quasilinearization technique due to Lakshmikantham [9] is applied to obtain an analytic approximation of the solution of the problem (1.1-1.2). In fact, we obtain sequences of upper and lower solutions converging monotonically and quadratically to a unique solution of the problem at hand. It is worth mentioning that the forced Duffing equation with mixed nonlinearities has not been studied so far.

\section{Preliminaries}

As argued in [12], the solution $x(t)$ of the problem (1.1-1.2) can be written in terms of the Green's function as

$$
\begin{aligned}
x(t) & =g_{1}(x(\sigma))\left(\frac{(p-q \lambda) e^{-\lambda}-p e^{-\lambda t}}{p\left[(p-q \lambda) e^{-\lambda}-(p+q \lambda)\right]}\right) \\
& +g_{2}(x(\sigma))\left(\frac{p e^{-\lambda t}-(p+q \lambda)}{p\left[(p-\lambda q) e^{-\lambda}-(p+\lambda q)\right]}\right)+\int_{0}^{1} G(t, s) N(s, x(s)) d s,
\end{aligned}
$$

where

$$
G(t, s)=\frac{p e^{\lambda s}}{\lambda\left[(p+q \lambda) e^{\lambda}-(p-q \lambda)\right]}\left\{\begin{array}{lll}
\left(e^{\lambda(1-s)}-\frac{(p-q \lambda)}{p}\right)\left(e^{-\lambda t}-\frac{(p+q \lambda)}{p}\right), & \text { if } & 0 \leq t \leq s \leq 1, \\
\left(e^{\lambda(1-t)}-\frac{(p-q \lambda)}{p}\right)\left(e^{-\lambda s}-\frac{(p+q \lambda)}{p}\right), & \text { if } & 0 \leq s \leq t \leq 1 .
\end{array}\right.
$$

Observe that $G(t, s)<0$ on $[0,1] \times[0,1]$.

Definition 2.1. We say that $\alpha \in C^{2}[J, \mathbb{R}]$ is a lower solution of the problem (1.1-1.2) if

$$
\begin{aligned}
& \alpha^{\prime \prime}(t)+\lambda \alpha^{\prime}(t) \geq N(t, \alpha), t \in J, \\
& p \alpha(0)-q \alpha^{\prime}(0) \leq g_{1}(\alpha(\sigma)), \quad p \alpha(1)+q \alpha^{\prime}(1) \leq g_{2}(\alpha(\sigma)),
\end{aligned}
$$

and $\beta \in C^{2}[$, $\mathbb{R}]$ will be an upper solution of the problem (1.1-1.2) if the inequalities are reversed in the definition of lower solution.

Now we state some basic results that play a pivotal role in the proof of the main result. We do not provide the proof as the method of proof is similar to the one described in the text [9].

Theorem 2.1. Let $\alpha$ and $\beta$ be lower and upper solutions of (1.1-1.2), respectively. Assume that

(i) $f_{x}(t, x)+k_{x}(t, x)-L>0$ for every $(t, x) \in J \times \mathbb{R}$.

(ii) $g_{1}$ and $g_{2}$ are continuous on $\mathbb{R}$ satisfying the one-sided Lipschitz condition:

$$
g_{i}(x)-g_{i}(y) \leq L_{i}(x-y), \quad 0 \leq L_{i}<1, \quad i=1,2 .
$$

Then $\alpha(t) \leq \beta(t), t \in J$.

Theorem 2.2. Let $\alpha$ and $\beta$ be lower and upper solutions of (1.1-1.2), respectively, such that $\alpha(t) \leq \beta(t), t \in J$. Then, there exists a solution $x(t)$ of (1.1-1.2) such that $\alpha(t)$ $\leq x(t) \leq \beta(t), t \in J$.

\section{Main result}

Theorem 3.1. Assume that

$\left(\mathbf{A}_{1}\right) \alpha_{0}, \beta_{0} \in C^{2}[J, \mathbb{R}]$ are lower and upper solutions of (1.1-1.2), respectively. 
$\left(\mathbf{A}_{2}\right) N \in C[\times \mathbb{R}, \mathbb{R}]$ be such that

$$
N(t, x)=f(t, x)+k(t, x)+H(t, x),
$$

where $f_{x}(t, x), k_{x}(t, x), f_{x x}(t, x), k_{x x}(t, x)$ exist and are continuous, and for continuous functions $\varphi, \chi,\left(f_{x x}(t, x)+\varphi_{x x}(t, x)\right) \geq 0,\left(k_{x x}(t, x)+\chi_{x x}(t, x)\right) \leq 0$ with $\varphi_{x x} \geq 0, \chi_{x x} \leq$ 0 for every $(t, x) \in S$, where $S=\left\{(t, x) \in J \times \mathbb{R}: \alpha_{0}(t) \leq x(t) \leq \beta_{0}(t)\right\} . H(t, x)$ satisfies the one-sided Lipschitz condition:

$$
H(t, x)-H(t, y) \geq-L(x-y), x \geq y, x, y \in \mathbb{R},
$$

where $L>0$ is a Lipschitz constant and $f_{x}(t, x)+k_{x}(t, x)-L>0$ for every $(t, x) \in S$.

$\left(\mathbf{A}_{3}\right)$ For $i=1,2, g_{i}, g_{i}^{\prime}, g_{i}^{\prime \prime}$ are continuous on $\mathbb{R}$ satisfying $0 \leq g_{i}^{\prime} \leq 1$ and $\left(g_{i}^{\prime \prime}(x)+\psi_{i}^{\prime \prime}(x)\right) \leq 0$ with $\psi_{i}^{i i} \leq 0$ on $\mathbb{R}$ for some continuous functions $\psi_{i}(x)$.

Then, there exist monotone sequences $\left\{\alpha_{n}\right\}$ and $\left\{\beta_{n}\right\}$ that converge in the space of continuous functions on $J$ quadratically to a unique solution $x(t)$ of the problem (1.11.2).

Proof. Let us define $F: J \times \mathbb{R} \rightarrow \mathbb{R}$ by $F(t, x)=f(t, x)+\varphi(t, x), K: J \times \mathbb{R} \rightarrow \mathbb{R}$ by $K(t$, $x)=k(t, x)+\chi(t, x), G_{i}: \mathbb{R} \rightarrow \mathbb{R}$ by $G_{i}(x)=g_{i}(x)+\psi_{i}(x), i=1,2$. By the assumption $\left(A_{2}\right)$ and the generalized mean value theorem, we get

$$
\begin{aligned}
& f(t, x) \geq f(t, y)+F_{x}(t, y)(x-y)-\phi(t, x)+\phi(t, y) . \\
& k(t, x) \geq k(t, y)+K_{x}(t, x)(x-y)+\psi(t, y)-\psi(t, x),
\end{aligned}
$$

Interchanging $x$ and $y,(3.1)$ and (3.2) take the form

$$
\begin{aligned}
& f(t, x) \leq f(t, y)+F_{x}(t, x)(x-y)-\phi(t, x)+\phi(t, y), \\
& k(t, x) \leq k(t, y)+K_{x}(t, y)(x-y)-\chi(t, x)+\chi(t, y) .
\end{aligned}
$$

By the assumption $\left(A_{3}\right)$, we obtain

$$
g_{i}(x) \geq g_{i}(y)+G_{i}^{\prime}(x)(x-y)+\psi_{i}(y)-\psi_{i}(x), \quad i=1,2,
$$

which, on interchanging $x$ and $y$ yields

$$
g_{i}(x) \leq g_{i}(y)+G_{i}^{\prime}(y)(x-y)+\psi_{i}(y)-\psi_{i}(x), \quad i=1,2 .
$$

We set

$$
\begin{aligned}
A\left(t, x ; \alpha_{0}, \beta_{0}\right) & =f\left(t, \alpha_{0}\right)+k\left(t, \alpha_{0}\right)+H(t, x) \\
& +\left[F_{x}\left(t, \beta_{0}\right)+K_{x}\left(t, \alpha_{0}\right)-\phi_{x}\left(t, \alpha_{0}\right)-\chi_{x}\left(t, \beta_{0}\right)\right]\left(x-\alpha_{0}\right), \\
B\left(t, x ; \alpha_{0}, \beta_{0}\right) & =f\left(t, \beta_{0}\right)+k\left(t, \beta_{0}\right)+H(t, x) \\
& +\left[F_{x}\left(t, \beta_{0}\right)+K_{x}\left(t, \alpha_{0}\right)-\phi_{x}\left(t, \alpha_{0}\right)-\chi_{x}\left(t, \beta_{0}\right)\right]\left(x-\beta_{0}\right),
\end{aligned}
$$


and for $i=1,2$,

$$
\begin{aligned}
h_{i}\left(x(\sigma) ; \alpha_{0}, \beta_{0}\right) & =g_{i}\left(\alpha_{0}(\sigma)\right)+G_{i}^{\prime}\left(\beta_{0}(\sigma)\right)\left(x(\sigma)-\alpha_{0}(\sigma)\right)+\psi_{i}\left(\alpha_{0}(\sigma)\right)-\psi_{i}(x(\sigma)), \\
\hat{h}_{i}\left(x(\sigma) ; \beta_{0}\right) & =g_{i}\left(\beta_{0}(\sigma)\right)+G_{i}^{\prime}\left(\beta_{0}(\sigma)\right)\left(x(\sigma)-\beta_{0}(\sigma)\right)+\psi_{i}\left(\beta_{0}(\sigma)\right)-\psi_{i}(x(\sigma)) .
\end{aligned}
$$

Observe that

$$
\begin{array}{ll}
A\left(t, \alpha_{0} ; \alpha_{0}, \beta_{0}\right)=N\left(t, \alpha_{0}\right), & N(t, x) \leq A\left(t, x ; \alpha_{0}, \beta_{0}\right), \\
h_{i}\left(\alpha_{0}(\sigma) ; \alpha_{0}, \beta_{0}\right)=g_{i}\left(\alpha_{0}(\sigma)\right), & g_{i}(x) \geq h_{i}\left(x(\sigma) ; \alpha_{0}, \beta_{0}\right), i=1,2,
\end{array}
$$

and

$$
\begin{array}{ll}
B\left(t, \beta_{0} ; \alpha_{0}, \beta_{0}\right)=N\left(t, \beta_{0}\right), & N(t, x) \geq B\left(t, x ; \alpha_{0}, \beta_{0}\right), \\
\hat{h}_{i}\left(\beta_{0}(\sigma) ; \beta_{0}\right)=g_{i}\left(\beta_{0}(\sigma)\right), & g_{i}(x) \leq \hat{h}_{i}\left(x(\sigma) ; \beta_{0}\right), i=1,2 .
\end{array}
$$

Now, we consider the problem

$$
\begin{aligned}
& x^{\prime \prime}(t)+\lambda x^{\prime}(t)=A\left(t, x ; \alpha_{0}, \beta_{0}\right), \quad t \in J, \\
& p x(0)-q x^{\prime}(0)=h_{1}\left(x(\sigma) ; \alpha_{0}, \beta_{0}\right), \quad p x(1)+q x^{\prime}(1)=h_{2}\left(x(\sigma) ; \alpha_{0}, \beta_{0}\right) .
\end{aligned}
$$

Using $\left(A_{1}\right)$, (3.7) and (3.8), we obtain

$$
\begin{aligned}
\alpha_{0}^{\prime \prime}(t)+\lambda \alpha_{0}^{\prime}(t) & \geq N\left(t, \alpha_{0}(t)\right)=A\left(t, \alpha_{0} ; \alpha_{0}, \beta_{0}\right), \\
p \alpha_{0}(0)-q \alpha_{0}^{\prime}(0) & \leq g_{1}\left(\alpha_{0}(\sigma)\right)=h_{1}\left(\alpha_{0}(\sigma) ; \alpha_{0}, \beta_{0}\right), \\
p \alpha_{0}(1)+q \alpha_{0}^{\prime}(1) & \leq g_{2}\left(\alpha_{0}(\sigma)\right)=h_{2}\left(\alpha_{0}(\sigma) ; \alpha_{0}, \beta_{0}\right),
\end{aligned}
$$

and

$$
\begin{aligned}
\beta_{0}^{\prime \prime}(t)+\lambda \beta_{0}^{\prime} & \leq N\left(t, \beta_{0}(t)\right) \leq A\left(t, \beta_{0} ; \beta_{0}, \beta_{0}\right), \\
p \beta_{0}(0)-q \beta_{0}^{\prime}(0) & \geq g_{1}\left(\beta_{0}(\sigma)\right) \geq h_{1}\left(\beta_{0}(\sigma) ; \alpha_{0}, \beta_{0}\right), \\
p \beta_{0}(1)+q \beta_{0}^{\prime}(1) & \geq g_{2}\left(\beta_{0}(\sigma)\right) \geq h_{2}\left(\beta_{0}(\sigma) ; \alpha_{0}, \beta_{0}\right),
\end{aligned}
$$

which imply that $\alpha_{0}$ and $\beta_{0}$ are, respectively, lower and upper solutions of (3.113.12). Thus, by Theorems 2.1 and 2.2, there exists a solution $\alpha_{1}$ for the problem (3.113.12) such that

$$
\alpha_{0}(t) \leq \alpha_{1}(t) \leq \beta_{0}(t), \quad t \in J .
$$

Next, consider the problem

$$
\begin{aligned}
& x^{\prime \prime}(t)+\lambda x^{\prime}(t)=B\left(t, x ; \alpha_{0}, \beta_{0}\right), \quad t \in J, \\
& p x(0)-q x^{\prime}(0)=\hat{h}_{1}\left(x(\sigma) ; \beta_{0}\right), \quad p x(1)+q x^{\prime}(1)=\hat{h}_{2}\left(x(\sigma) ; \beta_{0}\right) .
\end{aligned}
$$

Using $\left(A_{1}\right),(3.9)$ and (3.10), we get

$$
\begin{aligned}
\alpha_{0}^{\prime \prime}(t)+\lambda \alpha_{0}^{\prime}(t) & \geq N\left(t, \alpha_{0}(t)\right) \geq B\left(t, \alpha_{0} ; \alpha_{0}, \beta_{0}\right), \\
p \alpha_{0}(0)-q \alpha_{0}^{\prime}(0) & \leq g_{1}\left(\alpha_{0}(\sigma)\right) \leq \hat{h}_{1}\left(\alpha_{0}(\sigma) ; \beta_{0}\right), \\
p \alpha_{0}(1)+q \alpha_{0}^{\prime}(1) & \leq g_{2}\left(\alpha_{0}(\sigma)\right) \leq \hat{h}_{2}\left(\alpha_{0}(\sigma) ; \beta_{0}\right),
\end{aligned}
$$


and

$$
\begin{aligned}
\beta_{0}^{\prime \prime}(t)+\lambda \beta_{0}^{\prime} & \leq N\left(t, \beta_{0}(t)\right)=B\left(t, \beta_{0} ; \alpha_{0}, \beta_{0}\right), \\
p \beta_{0}(0)-q \beta_{0}^{\prime}(0) & \geq g_{1}\left(\beta_{0}(\sigma)\right)=\hat{h}_{1}\left(\beta_{0}(\sigma) ; \beta_{0}\right), \\
p \beta_{0}(1)+q \beta_{0}^{\prime}(1) & \geq g_{2}\left(\beta_{0}(\sigma)\right)=\hat{h}_{2}\left(\beta_{0}(\sigma) ; \beta_{0}\right),
\end{aligned}
$$

which imply that $\alpha_{0}$ and $\beta_{0}$ are, respectively, lower and upper solutions of (3.143.15). Again, by Theorems 2.1 and 2.2, there exists a solution $\beta_{1}$ of (3.14-3.15) satisfying

$$
\alpha_{0}(t) \leq \beta_{1}(t) \leq \beta_{0}(t), \quad t \in J .
$$

Now we show that $\alpha_{1}(t) \leq \beta_{1}(t)$. For that, we prove that $\alpha_{1}(t)$ is a lower solution and $\beta_{1}(t)$ is an upper solution of (1.1-1.2). Using the fact that $\alpha_{1}(t)$ is a solution of (3.113.12) satisfying $\alpha_{0}(t) \leq \alpha_{1}(t) \leq \beta_{0}(t)$ and (3.7-3.8), we obtain

$$
\begin{aligned}
& \alpha_{1}^{\prime \prime}(t)+\lambda \alpha_{1}^{\prime}(t)=A\left(t, \alpha_{1} ; \alpha_{0}, \beta_{0}\right) \geq N\left(t, \alpha_{1}(t)\right), \\
& p \alpha_{1}(0)-q \alpha_{1}^{\prime}(0)=h_{1}\left(\alpha_{1}(\sigma) ; \alpha_{0}, \beta_{0}\right) \leq g_{1}\left(\alpha_{1}(\sigma)\right), \\
& p \alpha_{1}(1)+q \alpha_{1}^{\prime}(1)=h_{2}\left(\alpha_{1}(\sigma) ; \alpha_{0}, \beta_{0}\right) \leq g_{2}\left(\alpha_{1}(\sigma)\right) .
\end{aligned}
$$

By the above inequalities, it follows that $\alpha_{1}$ is a lower solution of (1.1-1.2).

In view of the fact that $\beta_{1}(t)$ is a solution of (3.14-3.15) together with (3.9), we get

$$
\beta_{1}^{\prime \prime}(t)+\lambda \beta_{1}^{\prime}(t)=B\left(t, \beta_{1} ; \alpha_{0}, \beta_{0}\right) \leq N\left(t, \beta_{1}(t)\right),
$$

and by virtue of (3.10), we have

$$
\begin{aligned}
& p \beta_{1}(0)-q \beta_{1}^{\prime}(0)=\hat{h}_{1}\left(\beta_{1}(\sigma) ; \beta_{0}\right) \geq g_{1}\left(\beta_{1}(\sigma)\right), \\
& p \beta_{1}(1)+q \beta_{1}^{\prime}(1)=\hat{h}_{2}\left(\beta_{1}(\sigma) ; \beta_{0}\right) \geq g_{2}\left(\beta_{1}(\sigma)\right) .
\end{aligned}
$$

Thus, $\beta_{1}$ is an upper solution of (1.1-1.2). Hence, by Theorem 2.1, it follows that

$$
\alpha_{1}(t) \leq \beta_{1}(t), \quad t \in J .
$$

Combining $(3.13,3.16)$ and $(3.17)$ yields

$$
\alpha_{0}(t) \leq \alpha_{1}(t) \leq \beta_{1}(t) \leq \beta_{0}(t), \quad t \in J .
$$

Now, by induction, we prove that

$$
\alpha_{0}(t) \leq \alpha_{1}(t) \leq \cdots \leq \alpha_{n}(t) \leq \alpha_{n+1}(t) \leq \beta_{n+1}(t) \leq \beta_{n}(t) \leq \cdots \leq \beta_{1}(t) \leq \beta_{0}(t) .
$$

For that, we consider the boundary value problems

$$
\begin{aligned}
& x^{\prime \prime}(t)+\lambda x^{\prime}(t)=A\left(t, x ; \alpha_{n}, \beta_{n}\right), \quad t \in J, \\
& p x(0)-q x^{\prime}(0)=h_{1}\left(x(\sigma) ; \alpha_{n}, \beta_{n}\right), \quad p x(1)+q x^{\prime}(1)=h_{2}\left(x(\sigma) ; \alpha_{n}, \beta_{n}\right),
\end{aligned}
$$

and

$$
x^{\prime \prime}(t)+\lambda x^{\prime}(t)=B\left(t, x ; \alpha_{n}, \beta_{n}\right), \quad t \in J,
$$




$$
p x(0)-q x^{\prime}(0)=\hat{h}_{1}\left(x(\sigma) ; \beta_{n}\right), \quad \quad p x(1)+q x^{\prime}(1)=\hat{h}_{2}\left(x(\sigma) ; \beta_{n}\right) .
$$

Assume that for some $n>1, \alpha_{0}(t) \leq \alpha_{n}(t) \leq \beta_{n}(t) \leq \beta_{0}(t)$ and we will show that $\alpha_{n+1}$ $(t) \leq \beta_{n+1}(t)$.

Using (3.7), we have

$$
\alpha_{n}^{\prime \prime}(t)+\lambda \alpha_{n}^{\prime}(t)=A\left(t, \alpha_{n} ; \alpha_{n-1}, \beta_{n-1}\right) \geq N\left(t, \alpha_{n}\right)=A\left(t, \alpha_{n} ; \alpha_{n}, \beta_{n}\right) .
$$

By (3.8), we obtain

$$
h_{i}\left(\alpha_{n}(\sigma) ; \alpha_{n-1}, \beta_{n-1}\right) \leq g_{i}\left(\alpha_{n}(\sigma)\right)=h_{i}\left(\alpha_{n}(\sigma) ; \alpha_{n}, \beta_{n}\right),
$$

which yields

$$
p \alpha_{n}(0)-q \alpha_{n}^{\prime}(0) \leq h_{1}\left(\alpha_{n}(\sigma) ; \alpha_{n}, \beta_{n}\right), \quad p \alpha_{n}(1)+q \alpha_{n}^{\prime}(1) \leq h_{2}\left(\alpha_{n}(\sigma) ; \alpha_{n}, \beta_{n}\right) .
$$

Thus, $\alpha_{n}$ is a lower solution of (3.18-3.19). In a similar manner, we find that $\beta_{n}$ is an upper solution of (3.18-3.19). Thus, by Theorems 2.1 and 2.2, there exists a solution $\alpha_{n+1}(t)$ of (3.18-3.19) such that $\alpha_{n}(t) \leq \alpha_{n+1}(t) \leq \beta_{n}(t), t \in J$. Similarly, it can be proved that $\alpha_{n}(t) \leq \beta_{n+1}(t) \leq \beta_{n}(t), t \in J$, where $\beta_{n+1}(t)$ is a solution of (3.20-3.21) and $\alpha_{n}(t), \beta_{n}$ $(t)$ are lower and upper solutions of (3.20-3.21), respectively. Next, we show that $\alpha_{n+1}$ $(t) \leq \beta_{n+1}(t)$.

For that, we have to show that $\alpha_{n+1}(t)$ and $\beta_{n+1}(t)$ are lower and upper solutions of (1.1-1.2), respectively. Using $(3.7,3.8)$ together with the fact that $\alpha_{n+1}(t)$ is a solution of (3.18-3.19), we get

$$
\begin{aligned}
& \alpha_{n+1}^{\prime \prime}(t)+\lambda \alpha_{n+1}^{\prime}(t)=A\left(t, \alpha_{n+1} ; \alpha_{n}, \beta_{n}\right) \geq N\left(t, \alpha_{n+1}\right), \\
& p \alpha_{n+1}(0)-q \alpha_{n+1}^{\prime}(0)=h_{i}\left(\alpha_{n+1}(\sigma) ; \alpha_{n}, \beta_{n}\right) \leq g_{1}\left(\alpha_{n+1}(\sigma)\right), \\
& p \alpha_{n+1}(1)+q \alpha_{n+1}^{\prime}(1)=h_{i}\left(\alpha_{n+1}(\sigma) ; \alpha_{n}, \beta_{n}\right) \leq g_{2}\left(\alpha_{n+1}(\sigma)\right),
\end{aligned}
$$

which implies that $\alpha_{n+1}$ is a lower solution of (1.1-1.2). Employing a similar procedure, it can be proved that $\beta_{n+1}$ is an upper solution of (1.1-1.2). Hence, by Theorem 2.1, it follows that $\alpha_{n+1}(t) \leq \beta_{n+1}(t)$. Therefore, by induction, we have

$$
\alpha_{0}(t) \leq \alpha_{1}(t) \leq \cdots \leq \alpha_{n}(t) \leq \alpha_{n+1}(t) \leq \beta_{n+1}(t) \leq \beta_{n}(t) \leq \cdots \leq \beta_{1}(t) \leq \beta_{0}(t), \forall n \in \mathbb{N} .
$$

Since $[0,1]$ is compact and the monotone convergence is pointwise, it follows that $\left\{\alpha_{n}\right\}$ and $\left\{\beta_{n}\right\}$ are uniformly convergent with

$$
\lim _{n \rightarrow \infty} \alpha_{n}(t)=x(t), \quad \quad \lim _{n \rightarrow \infty} \beta_{n}(t)=\gamma(t),
$$

such that $\alpha_{0}(t) \leq x(t) \leq y(t) \leq \beta_{0}(t)$, where

$$
\begin{aligned}
\alpha_{n}(t) & =h_{1}\left(\alpha_{n}(\sigma) ; \alpha_{n-1}, \beta_{n-1}\right) \frac{(p-q \lambda) e^{-\lambda}-p e^{-\lambda t}}{p\left[(p-q \lambda) e^{-\lambda}-(p+q \lambda)\right]} \\
& +h_{2}\left(\alpha_{n}(\sigma) ; \alpha_{n-1}, \beta_{n-1}\right) \frac{(p+q \lambda)-p e^{-\lambda t}}{p\left[(p+\lambda q)-(p-\lambda q) e^{-\lambda}\right]} \\
& +\int_{0}^{1} G(t, s) A\left(s, \alpha_{n}(s) ; \alpha_{n-1}, \beta_{n-1}\right) d s,
\end{aligned}
$$


and

$$
\begin{aligned}
\beta_{n}(t) & =\hat{h}_{1}\left(\beta_{n}(\sigma) ; \beta_{n-1}\right) \frac{(p-q \lambda) e^{-\lambda}-p e^{-\lambda t}}{p\left[(p-q \lambda) e^{-\lambda}-(p+q \lambda)\right]} \\
& +\hat{h}_{2}\left(\beta_{n}(\sigma) ; \beta_{n-1}\right) \frac{(p+q \lambda)-p e^{-\lambda t}}{p\left[(p+\lambda q)-(p-\lambda q) e^{-\lambda}\right]} \\
& +\int_{0}^{1} G(t, s) B\left(s, \beta_{n}(s) ; \beta_{n-1}, \beta_{n-1}\right) d s .
\end{aligned}
$$

By the uniqueness of the solution (which follows by the hypotheses of Theorem 2.1), we conclude that $x(t)=y(t)$. This proves that the problem (1.1-1.2) has a unique solution $x(t)$ given by

$$
\begin{gathered}
x(t)=g_{1}(x(\sigma)) \frac{(p-q \lambda) e^{-\lambda}-p e^{-\lambda t}}{p\left[(p-q \lambda) e^{-\lambda}-(p+q \lambda)\right]}+g_{2}(x(\sigma)) \frac{(p+q \lambda)-p e^{-\lambda t}}{p\left[(p+\lambda q)-(p-\lambda q) e^{-\lambda}\right]} \\
+\int_{0}^{1} G(t, s) N(s, x(s)) \mathrm{d} s .
\end{gathered}
$$

In order to prove that each of the sequences $\left\{\alpha_{n}\right\},\left\{\beta_{n}\right\}$ converges quadratically, we set $z_{n}(t)=\beta_{n}(t)-x(t)$ and $r_{n}(t)=x(t)-\alpha_{n}(t)$, and note that $z_{n} \geq 0, r_{n} \geq 0$. We will only prove the quadratic convergence of the sequence $\left\{r_{n}\right\}$ as that of $\left\{z_{n}\right\}$ is similar. By the mean value theorem, we find that

$$
\begin{aligned}
& r_{n+1}^{\prime \prime}(t)+\lambda r_{n+1}^{\prime}(t) \\
= & x^{\prime \prime}(t)-\alpha_{n+1}^{\prime \prime}(t)+\lambda\left[x^{\prime}(t)-\alpha_{n+1}^{\prime}(t)\right] \\
= & {\left.\left[x^{\prime \prime}(t)+\lambda x^{\prime}(t)\right]-\left[\alpha_{n+1}^{\prime \prime}(t)+\lambda \alpha_{n+1}^{\prime}(t)\right)\right] } \\
= & N(t, x)-A\left(t, \alpha_{n+1}, \alpha_{n}, \beta_{n}\right) \\
= & F(t, x)+K(t, x)+H(t, x)-\phi(t, x)-\chi(t, x)-F\left(t, \alpha_{n}\right) \\
- & K\left(t, \alpha_{n}\right)-H\left(t, \alpha_{n+1}\right)+\phi\left(t, \alpha_{n}\right)+\chi\left(t, \alpha_{n}\right) \\
- & {\left[F_{x}\left(t, \beta_{n}\right)+K_{x}\left(t, \alpha_{n}\right)-\phi_{x}\left(t, \alpha_{n}\right)-\chi_{x}\left(t, \beta_{n}\right)\right]\left(\alpha_{n+1}-\alpha_{n}\right) } \\
= & F(t, x)+K(t, x)+H(t, x)-\phi(t, x)-\chi(t, x)-F\left(t, \alpha_{n}\right) \\
- & K\left(t, \alpha_{n}\right)-H\left(t, \alpha_{n+1}\right)+\phi\left(t, \alpha_{n}\right)+\chi\left(t, \alpha_{n}\right) \\
- & {\left[F_{x}\left(t, \alpha_{n}\right)+K_{x}\left(t, \alpha_{n}\right)-\phi_{x}\left(t, \alpha_{n}\right)-\chi_{x}\left(t, \beta_{n}\right)\right]\left(r_{n}-r_{n+1}\right) } \\
\geq & F_{x}\left(t, \xi_{1}\right) r_{n}+K_{x}\left(t, \xi_{2}\right) r_{n}-L r_{n+1}-\phi_{x}\left(t, \xi_{3}\right) r_{n}-\chi_{x}\left(t, \xi_{4}\right) r_{n} \\
- & {\left[F_{x}\left(t, \beta_{n}\right)+K_{x}\left(t, \alpha_{n}\right)-\phi_{x}\left(t, \alpha_{n}\right)-\chi_{x}\left(t, \beta_{n}\right)\right]\left(r_{n}-r_{n+1}\right) } \\
\geq & {\left[F_{x}\left(t, \alpha_{n}\right)-F_{x}\left(t, \beta_{n}\right)\right] r_{n}+\left[K_{x}(t, x)-K_{x}\left(t, \alpha_{n}\right)\right] r_{n} } \\
- & {\left[\phi_{x}(t, x)-\phi_{x}\left(t, \alpha_{n}\right)\right] r_{n}+\left[\chi_{x}\left(t, \beta_{n}\right)-\chi_{x}\left(t, \alpha_{n}\right)\right] r_{n} } \\
+ & {\left[-L+F_{x}\left(t, \beta_{n}\right)+K_{x}\left(t, \alpha_{n}\right)-\phi_{x}\left(t, \alpha_{n}\right)-\chi_{x}\left(t, \beta_{n}\right)\right] r_{n+1} } \\
\geq & {\left[-F_{x x}\left(t, \zeta_{5}\right)+\chi_{x x}(t, \zeta 8)\right] r_{n}\left(\beta_{n}-\alpha_{n}\right)+K_{x x}(t, \zeta 6) r_{n}^{2}-\phi_{x x}\left(t, \zeta_{7}\right) r_{n}^{2} } \\
+ & {\left[-L+F_{x}\left(t, \alpha_{n}\right)+K_{x}\left(t, \alpha_{n}\right)-\phi_{x}\left(t, \alpha_{n}\right)-\chi_{x}\left(t, \alpha_{n}\right)\right] r_{n+1} } \\
\geq & {\left[-F_{x x}\left(t, \zeta_{5}\right)+\chi_{x x}(t, \zeta 8)\right] r_{n}\left(z_{n}+r_{n}\right)+K_{x x}\left(t, \zeta_{6}\right) r_{n}^{2}-\phi_{x x}(t, \zeta 7) r_{n}^{2} } \\
\geq & {\left[F_{x x}\left(t, \zeta_{5}\right)-\chi_{x x}\left(t, \zeta_{8}\right)\right]\left(\frac{-3}{2} r_{n}^{2}-\frac{1}{2} z_{n}^{2}\right)+\left[K_{x x}\left(t, \zeta_{6}\right)-\phi_{x x}\left(t, \zeta_{7}\right)\right] r_{n}^{2} } \\
\geq & {\left[\frac{-3}{2} F_{x x}\left(t, \zeta_{5}\right)+\frac{3}{2} \chi_{x x}\left(t, \zeta_{8}\right)+K_{x x}\left(t, \zeta_{6}\right)-\phi_{x x}\left(t, \zeta_{7}\right)\right] r_{n}^{2} } \\
+ & {\left[\frac{-1}{2} F_{x x}\left(t, \zeta_{5}\right)+\frac{1}{2} \chi_{x x}(t, \zeta 8)\right] z_{n}^{2} } \\
\geq & \left.-\left[\frac{3}{2} C_{1}+\frac{3}{2} C_{4}+C_{2}+C_{3}\right]\left\|r_{n}\right\|^{2}+M_{2}\|\|_{n} \|^{2}\right), \frac{1}{2}\left[C_{1}+C_{4}\right]\left\|z_{n}\right\|^{2} \\
&
\end{aligned}
$$


where $\alpha_{n} \leq \zeta_{5}, \quad \zeta_{8} \leq \beta_{n}, \alpha_{n} \leq \zeta_{6}, \zeta_{7} \leq x$, and $\left|F_{x x}\right| \leq C_{1},\left|K_{x x}\right| \leq C_{2},\left|\phi_{x x}\right| \leq C_{3},\left|\chi_{x x}\right| \leq C_{4}, M_{1}=\frac{3}{2} C_{1}+\frac{3}{2} C_{4}+C_{2}+C_{3} \quad$ and $M_{2}=\frac{1}{2}\left(C_{1}+C_{2}\right)$.

Now we define

$$
N_{1}(t)=\frac{(p-q \lambda) e^{-\lambda}-p e^{-\lambda t}}{p\left[(p-q \lambda) e^{-\lambda}-(p+q \lambda)\right]}, \quad N_{2}(t)=\frac{(p+q \lambda)-p e^{-\lambda t}}{p\left[(p+\lambda q)-(p-\lambda q) e^{-\lambda}\right]}
$$

and obtain

$$
\begin{aligned}
& r_{n+1}(t)=x(t)-\alpha_{n+1}(t) \\
& =N_{1}(t)\left[g_{1}(x(\sigma))-h_{1}\left(\alpha_{n+1}(\sigma) ; \alpha_{n}, \beta_{n}\right)\right]+N_{2}(t)\left[g_{2}(x(\sigma))-h_{2}\left(\alpha_{n+1}(\sigma) ; \alpha_{n}, \beta_{n}\right)\right] \\
& +\int_{0}^{1} G(t, s)\left[\left[N(s, x(s))-A\left(s, \alpha_{n+1}(s) ; \alpha_{n}, \beta_{n}\right)\right] d s\right. \\
& =N_{1}(t)\left[g_{1}(x(\sigma))-h_{1}\left(\alpha_{n+1}(\sigma) ; \alpha_{n}, \beta_{n}\right)\right]+N_{2}(t)\left[g_{2}(x(\sigma))-h_{2}\left(\alpha_{n+1}(\sigma) ; \alpha_{n}, \beta_{n}\right)\right] \\
& +\int_{0}^{1} G(t, s)\left[r_{n+1}^{\prime \prime}(s)+\lambda r_{n+1}^{\prime}(s)\right] d s \\
& \leq N_{1}(t)\left[g_{1}(x(\sigma))-g_{1}\left(\alpha_{n}(\sigma)\right)-G_{1}^{\prime}\left(\beta_{n}(\sigma)\right)\left(\alpha_{n+1}(\sigma)-\alpha_{n}(\sigma)\right)\right. \\
& \left.-\psi_{1}\left(\alpha_{n}(\sigma)\right)+\psi_{1}\left(\alpha_{n+1}(\sigma)\right)\right]+N_{2}(t)\left[g_{2}(x(\sigma))-g_{2}\left(\alpha_{n}(\sigma)\right)\right. \\
& \left.-G_{2}^{\prime}\left(\beta_{n}(\sigma)\right)\left(\alpha_{n+1}(\sigma)-\alpha_{n}(\sigma)\right)-\psi_{2}\left(\alpha_{n}(\sigma)\right)+\psi_{2}\left(\alpha_{n+1}(\sigma)\right)\right] \\
& +\left(M_{1}\left\|r_{n}\right\|^{2}+M_{2}\left\|z_{n}\right\|^{2}\right) \int_{0}^{1}|G(t, s)| d s \\
& \leq N_{1}(t)\left[g_{1}^{\prime}\left(\gamma_{1}\right) r_{n}-G_{1}^{\prime}\left(\beta_{n}(\sigma)\right)\left(r_{n}-r_{n+1}\right)+\psi_{1}^{\prime}\left(\gamma_{2}\right)\left(r_{n}-r_{n+1}\right)\right] \\
& +N_{2}(t)\left[g_{2}^{\prime}\left(\delta_{1}\right) r_{n}-G_{2}^{\prime}\left(\beta_{n}(\sigma)\right)\left(r_{n}-r_{n+1}\right)+\psi_{2}^{\prime}\left(\delta_{2}\right)\left(r_{n}-r_{n+1}\right)\right] \\
& +M_{0}\left(M_{1}\left\|r_{n}\right\|^{2}+M_{2}\left\|z_{n}\right\|^{2}\right) \text {. } \\
& \leq N_{1}(t)\left[G_{1}^{\prime}\left(\gamma_{1}\right) r_{n}-\psi_{1}^{\prime}\left(\gamma_{1}\right) r_{n}-G_{1}^{\prime}\left(\beta_{n}(\sigma)\right) r_{n}+G_{1}^{\prime}\left(\beta_{n}(\sigma)\right) r_{n+1}\right) \\
& \left.\left.+\psi_{1}^{\prime}\left(\gamma_{2}\right) r_{n}-\psi_{1}^{\prime}\left(\gamma_{2}\right) r_{n+1}\right)\right]+N_{2}(t)\left[G_{2}^{\prime}\left(\delta_{1}\right) r_{n}-\psi_{2}^{\prime}\left(\delta_{1}\right) r_{n}\right. \\
& \left.\left.\left.-G_{2}^{\prime}\left(\beta_{n}(\sigma)\right) r_{n}+G_{2}^{\prime}\left(\beta_{n}(\sigma)\right) r_{n+1}\right)+\psi_{2}^{\prime}\left(\delta_{2}\right) r_{n}-\psi_{2}^{\prime}\left(\delta_{2}\right) r_{n+1}\right)\right] \\
& +M_{0}\left(M_{1}\left\|r_{n}\right\|^{2}+M_{2}\left\|z_{n}\right\|^{2}\right) \text {. } \\
& \leq N_{1}(t)\left[\left(G_{1}^{\prime}\left(\alpha_{n}(\sigma)\right)-G_{1}^{\prime}\left(\beta_{n}(\sigma)\right)\right) r_{n}-\left(\psi_{1}^{\prime}(x(\sigma)) r_{n}-\psi_{1}^{\prime}\left(u_{n}(\sigma)\right) r_{n}\right)\right. \\
& +\left(G_{1}^{\prime}\left(\beta_{n}(\sigma)\right)-\psi_{1}^{\prime}\left(\alpha_{n}(\sigma)\right) r_{n+1}\right]+N_{2}(t)\left[G_{2}^{\prime}\left(\alpha_{n}(\sigma)\right) r_{n}-G_{2}^{\prime}\left(\beta_{n}(\sigma)\right) r_{n}\right. \\
& \left.\left.-\psi_{2}^{\prime}(x(\sigma)) r_{n}+\psi_{2}^{\prime}\left(\alpha_{n}(\sigma)\right) r_{n}+\left(G_{2}^{\prime}\left(\beta_{n}(\sigma)\right)-\psi_{2}^{\prime}\left(\alpha_{n}(\sigma)\right)\right) r_{n+1}\right)\right] \\
& +M_{0}\left(M_{1}\left\|r_{n}\right\|^{2}+M_{2}\left\|z_{n}\right\|^{2}\right) \\
& \leq N_{1}(t)\left[\left(-G_{1}^{\prime \prime}\left(\rho_{1}\right) r_{n}\left(z_{n}+r_{n}\right)-\psi_{1}^{\prime \prime}\left(\rho_{2}\right) r_{n}^{2}+g_{1}^{\prime}\left(\alpha_{n}(\sigma)\right) r_{n+1}\right]\right. \\
& \left.+N_{2}(t)\left[-G_{2}^{\prime \prime}\left(\sigma_{1}\right) r_{n}\left(z_{n}+r_{n}\right)-\psi_{2}^{\prime \prime}\left(\sigma_{2}\right) r_{n}^{2}+g_{2}^{\prime}\left(\alpha_{n}(\sigma)\right) r_{n+1}\right)\right] \\
& +M_{0}\left(M_{1}\left\|r_{n}\right\|^{2}+M_{2}\left\|z_{n}\right\|^{2}\right) \\
& \leq N_{1}(t)\left[\left(-G_{1}^{\prime \prime}\left(\rho_{1}\right)\left(\frac{3}{2} r_{n}^{2}+\frac{1}{2} z_{n}^{2}\right)-\psi_{1}^{\prime \prime}\left(\rho_{2}\right) r_{n}^{2}+r_{n+1}\right)\right]+N_{2}(t)\left[-G_{2}^{\prime \prime}\left(\sigma_{1}\right)\left(\frac{3}{2} r_{n}^{2}+\frac{1}{2} z_{n}^{2}\right)\right. \\
& \left.\left.-\psi_{2}^{\prime \prime}\left(\sigma_{2}\right) r_{n}^{2}+r_{n+1}\right)\right]+M_{0}\left(M_{1}\left\|r_{n}\right\|^{2}+M_{2}\left\|z_{n}\right\|^{2}\right) \text {. }
\end{aligned}
$$

where $\alpha_{n} \leq \gamma_{1}, \delta_{1}, \rho_{1}, \sigma_{1} \leq x, \alpha_{n} \leq \gamma_{2} \leq x$, and $\alpha_{n} \leq \delta_{2}, \rho_{2}, \sigma_{2} \leq \alpha_{n+1}$. Letting $\left|G_{i}^{\prime \prime}\right|<D_{i},\left|\psi_{i}^{\prime \prime}\right|<E_{i}, \max _{t \in[0,1]}\left|N_{i}\right|=\bar{N}_{i}(i=1,2)$ and $M_{0}$ as an upper bound on $M \int_{0}^{1} G(t, s) \mathrm{d} s$, we obtain

$$
\left\|r_{n+1}(t)\right\| \leq \frac{\left.\left\|r_{n}\right\|^{2} W_{1}+\left\|z_{n}\right\|^{2}\right) W_{2}}{(1-\eta)}
$$


where $\quad \eta=\left(\bar{N}_{1}+\bar{N}_{2}\right)<1, W_{1}=\left[\frac{3}{2} \bar{N}_{1} D_{1}+\bar{N}_{1} E_{1}+\frac{3}{2} \bar{N}_{2} D_{2} \bar{N}_{2} E_{2}+M_{0} M_{1}\right], \quad$ and $W_{2}=\left[+\frac{1}{2} \bar{N}_{1} D_{1}+\frac{1}{2} \bar{N}_{2} D_{2}+M_{0} M_{2}\right]$. This completes the proof.

\section{Examples}

Example 4.1. Consider the problem

$$
\begin{aligned}
& x^{\prime \prime}(t)+x^{\prime}(t)=2 x-t \cos (\pi x / 2), \\
& 3 x(0)-2 x^{\prime}(0)=\frac{1}{3} x(1 / 2)+1, \quad 3 x(1)+2 x^{\prime}(1)=\frac{1}{2} x(1 / 2)+2 .
\end{aligned}
$$

Here $f(t, \quad x)=2 x-t \cos (\pi x / 2), \quad k(t, \quad x) \equiv 0$, $H(t, x) \equiv 0, g_{1}\left(x\left(\frac{1}{2}\right)\right)=\frac{1}{3} x(1 / 2)+1, g_{2}\left(x\left(\frac{1}{2}\right)\right)=\frac{1}{2} x(1 / 2)+2$. Let $\alpha_{0}=0$ and $\beta_{0}$ $=1$ be lower and upper solutions of (4.1-4.2), respectively. We note that $f_{x}(t, x)=2-\frac{\pi}{2} t \sin (\pi x / 2)>0, g_{1}^{\prime}\left(x\left(\frac{1}{2}\right)\right)=1 / 3, g_{2}^{\prime}\left(x\left(\frac{1}{2}\right)\right)=1 / 2$. Further, we choose $\phi(t, x)=3 x^{2}, \psi_{i}(x)=-\hat{M}_{i}(x+1)^{2}, \hat{M}_{i}>0, i=1,2$. We note that $f_{x x}(t, x)+\phi_{x x}(t, x)=-\frac{\pi^{2}}{4} t \cos (\pi x / 2)+6 \geq 0, g_{i}^{\prime \prime}(x)+\psi_{i}^{\prime \prime}(x) \leq 0$. Thus, all the conditions of Theorem (3.1) are satisfied. Hence, the conclusion of Theorem 3.1 applies to the problem (4.1-4.2).

Example 4.2. Consider the nonlinear boundary value problem given by

$$
\begin{aligned}
& x^{\prime \prime}(t)+\lambda x^{\prime}(t)=7 x+\sin (\pi x t / 2)-t \cos (\pi x / 2)+\frac{1}{2}|x|, \quad t \in[0,1], \\
& 3 x(0)-2 x^{\prime}(0)=\frac{x(t)}{4}+1, \quad 3 x(1)+2 x^{\prime}(1)=\frac{x(t)}{2}+2,
\end{aligned}
$$

where $f(t, x)=7 x+\sin (\pi x t / 2), \quad k(t, x)=-t \cos (\pi x / 2)$, $H(t, x)=\frac{1}{2}|x|, L=\frac{1}{2}, g_{1}(x)=x(t) / 4+1, g_{2}(x)=x(t) / 2+2$. Let $\alpha_{0}=0$ and $\beta_{0}=1$ be lower and upper solutions of (4.1-4.2), respectively. Observe that

$$
f_{x}(t, x)+k_{x}(t, x)-\frac{1}{2}=7+\frac{t \pi}{2} \cos \frac{\pi}{2} x t+\frac{t \pi}{2} \sin \frac{\pi}{2} x-\frac{1}{2}>0,
$$

and $0 \leq g_{i}^{\prime}(x) \leq 1$. For positive constants $M_{1}, M_{2}, N_{1}, N_{2}$, we choose

$$
\phi(t, x)=M_{1} \frac{\pi^{2}}{4} t^{2}(1+x)^{2}, \chi(t, x)=-M_{2} \pi^{2} x^{2}, \quad \psi_{i}(x)=-N_{i}(x+2)^{2},
$$

such that $f_{x x}(t, x)+\varphi_{x x}(t, x)=\pi^{2} t^{2}\left[2 M_{1}-\cos (\pi t x / 2)\right] / 4 \geq 0, k_{x x}+\chi_{x x}=-\pi^{2}\left[8 M_{2}-\right.$ $t \cos (\pi x / 2)] / 4 \leq 0$. Clearly, $g_{i}^{\prime \prime}(x)+\psi_{i}^{\prime \prime}(x) \leq 0$. Thus, all the conditions of Theorem 3.1 are satisfied. Hence, the conclusion of theorem (3.1) applies to the problem (4.3-4.4). 


\section{Authors' contributions}

Both authors, $\mathrm{AA}$ and MHA, contributed to each part of this work equally and read and approved the final version of the manuscript.

\section{Competing interests}

The authors declare that they have no competing interests.

Received: 15 June 2011 Accepted: 25 November 2011 Published: 25 November 2011

\section{References}

1. Yolasi, CK, Kyprianidis, IM, Stovbouios, I: Experimental study of a nonlinear circuit described by Duffing's equation. J Istanbul Kültür Univ. 4, 45-54 (2006)

2. Vaidya, PG, Winkel, CR: Analysis of induced chaos in Duffing's equation, using Caseygrams. J Acoust Soc Am. 99(4), 2496-2500 (1996)

3. Ladde, GS, Lakshmikantham, V, Vatsala, AS: Monotone Iterative Techniques for Nonlinear Differential Equations. Pitman, Boston (1985)

4. Bellman, R, Kalaba, R: Quasilinearization and Nonlinear Boundary Value Problems. Amer Elsevier New York (1965)

5. Lee, ES: Quasilinearization and Invariant Embedding. Academic Press, New York (1968)

6. Agarwal, RP: Quasilinearization and approximate quasilinearization for multipoint boundary value problems. J Math Anal Appl. 107, 317-330 (1985). doi:10.1016/0022-247X(85)90372-5

7. Lakshmikantham, V: An extension of the method of quasilinearization. J Optim Theory Appl. 82, 315-321 (1994). do:10.1007/BF02191856

8. Lakshmikantham, V: Further improvement of generalized quasilinearization. Nonlinear Anal. 27, 223-227 (1996). doi:10.1016/0362-546X(94)00281-L

9. Lakshmikantham, V, Vatsala, AS: Generalized Quasilinearization for Nonlinear Problems, Mathematics and its Applications. Kluwer, Dordrecht440 (1998)

10. Ahmad, B, Nieto, JJ, Shahzad, N: The Bellman-Kalaba-Lakshmikantham quasilinearization method for Neumann problems. J Math Anal Appl. 257, 356-363 (2001). doi:10.1006/jmaa.2000.7352

11. Mandelzweig, VB, Tabakin, F: Quasilinearization approach to nonlinear problems in physics with application to nonlinear ODEs. Comput Phys Comm. 141, 268-281 (2001). doi:10.1016/S0010-4655(01)00415-5

12. Eloe, PW, Gao, Y: The method of quasilinearization and a three-point boundary value problem. J Korean Math Soc. 39 319-330 (2002)

13. Ramos, Jl: Piecewise quasilinearization techniques for singular boundary-value problems. Comput Phys Comm. 158 12-25 (2004). doi:10.1016/j.comphy.2003.11.003

14. Ahmad, B: A quasilinearization method for a class of integro-differential equations with mixed nonlinearities. Nonlinear Anal Real World Appl. 7, 997-1004 (2006). doi:10.1016/j.nonrwa.2005.09.003

15. Alsaedi, A: Monotone iteration scheme for a forced Duffing equation with nonlocal three-point conditions. Commun Korean Math Soc. 22(1), 53-64 (2007). doi:10.4134/CKMS.2007.22.1.053

16. Amster, P, De Napoli, P: A quasilinearization method for elliptic problems with a nonlinear boundary condition. Nonlinear Anal. 66, 2255-2263 (2007). doi:10.1016/j.na.2006.03.016

17. O'Regan, D, El-Gebeily, M: A quasilinearization method for a class of second order singular nonlinear differential equations with nonlinear boundary conditions. Nonlinear Anal Real World Appl. 8, 174-186 (2007). doi:10.1016/j. nonrwa.2005.06.008

18. Ahmad, B, Alsaedi, A, Alghamdi, B: Analytic approximation of solutions of the forced Duffing equation with integral boundary conditions. Nonlinear Anal Real World Appl. 9, 1727-1740 (2008). doi:10.1016/j.nonrwa.2007.05.005

19. Ahmad, B, Nieto, J: Existence and approximation of solutions for a class of nonlinear impulsive functional differential equations with anti-periodic boundary conditions. Nonlinear Anal. 69, 3291-3298 (2008). doi:10.1016/j.na.2007.09.018

20. Ahmad, B, Alghamdi, B: Approximation of solutions of the nonlinear Duffing equation involving both integral and nonintegral forcing terms with separated boundary conditions. Comput Phys Comm. 179, 409-416 (2008). doi:10.1016/j. срc.2008.04.008

21. Pei, M, Chang, SK: A quasilinearization method for second-order four-point boundary value problems. Appl Math Comp. 202, 54-66 (2008). doi:10.1016/j.amc.2008.01.026

22. Pei, M, Chang, SK: A quasilinearization method for second-order four-point boundary value problems. Appl Math Comp. 202, 54-66 (2008). doi:10.1016/j.amc.2008.01.026

23. O'Regan, D, El-Gebeily, M: Existence, upper and lower solutions and quasilinearization for singular differential equations. IMA J Appl Math. 73, 323-344 (2008)

24. Ahmad, B, Alsaedi, A: Existence of approximate solutions of the forced Duffing equation with discontinuous type integral boundary conditions. Nonlinear Anal Real World Appl. 10, 358-367 (2009). doi:10.1016/j.nonrwa.2007.09.004

25. Nieto, JJ, Ahmad, B: Approximation of solutions for an initial and terminal value problem for the forced Duffing equation with non-viscous damping. Appl Math Comput. 216, 2129-2136 (2010). doi:10.1016/j.amc.2010.03.046

26. Alsaedi, A: Approximation of solutions for second-order $m$-point nonlocal boundary value problems via the method of generalized quasilinearization. Bound Value Probl 17 (2011). (Art. ID 929061)

doi:10.1186/1687-2770-2011-47

Cite this article as: Alsaedi and Aqlan: On nonlocal three-point boundary value problems of Duffing equation with mixed nonlinear forcing terms. Boundary Value Problems 2011 2011:47. 